\title{
Supplementary Information: How Does Monomer Structure Affect the Interfacial Dynamics of Supported Ultrathin Polymer Films?
}

\author{
Amber Storey, ${ }^{\dagger}$, Wengang Zhang, ${ }^{*,+, \dagger, \uparrow}$ Jack F. Douglas, ${ }^{a *, \ddagger}$ and \\ Francis W. Starr ${ }^{*, \dagger}$ \\ $\dagger$ Department of Physics, Wesleyan University, Middletown, Connecticut 06459-0155, USA. \\ $\ddagger$ Materials Science and Engineering Division, Material Measurement Laboratory, National \\ Institute of Standards and Technology, Gaithersburg, Maryland 20899, USA. \\ 9A.N. Storey and W. Zhang contributed equally to this work. \\ E-mail: wzhang01@wesleyan.edu; jack.douglas@nist.gov; fstarr@wesleyan.edu
}

\footnotetext{
${ }^{a}$ Contribution of the National Institute of Standards and Technology - Work not subject to copyright in the United States
} 


\section{Additional Simulation Detail}

\section{Film System Preparation}

To create a polymer film with thickness $\approx 50 \AA$ at $T_{g}$, PEO films have $N=3000$ coarsegrained beads and PMMA film has $N=5000$ coarse-grained beads, excluding the substrate particles. We create a substrate with side lengths $\approx 20 \sigma$ for PMMA and $\approx 15 \sigma$ for PEO. To model a thin film, we apply periodic boundary conditions in the $x, y$-directions, parallel to the substrate, with the $z$ dimension left non-periodic to model a free surface (empty space) on top of the film creating an effective pressure of $\langle P\rangle=0$ atm. All film simulations are conducted at fixed NVT to keep the dimensions of the substrate lattice. The interaction between the top and bottom walls and the substrate is a 9-3 Lennard-Jones potential.

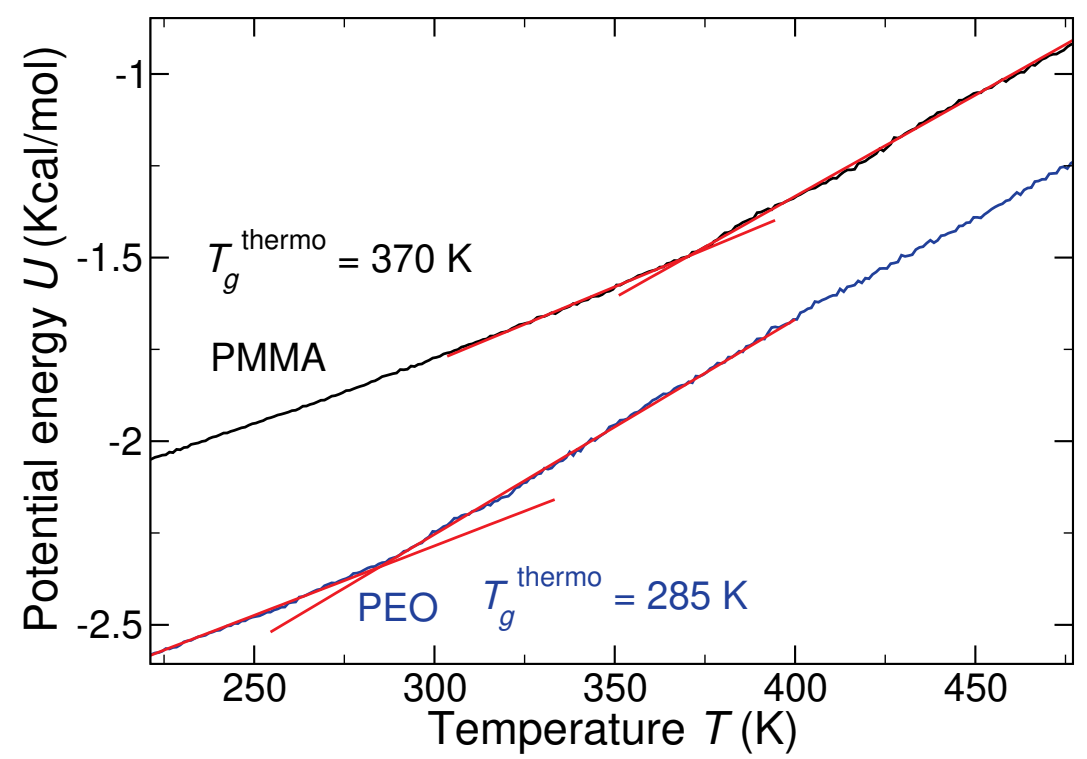

Figure S1: The temperature dependence of the potential energy for PMMA and PEO film supported by a $\mathrm{SiO}_{2} / \mathrm{Si}$ substrate. The heating rate is $10 \mathrm{~K} / \mathrm{ns}$ for both films. The potential energy of the PEO film is shifted upward by $2 \mathrm{Kcal} / \mathrm{mol}$ for the clarity of the figure. 


\section{PEO}

The Lennard-Jones (LJ) potential was applied for interactions between non-bonded monomers, meaning any two monomers not connected via a bond, defined as,

$$
V_{\text {non-bonded }}(r)=V_{L J}(r)+S(r)
$$

where $S(r)$ is a function that shifts the potential smoothly to zero between the designated cutoffs, $r_{1}=9.0 \AA$ and $r_{c}=12.0 \AA$. These cutoffs are used to designate when the function $S(r)$ should start (at $r_{1}$ ) and stop (at $r_{c}$ ) the shifting, where the potential at $r_{c}$ should be zero. $V_{L J}$ is defined as,

$$
V_{L J}(r)=4 \varepsilon_{i j}\left[\left(\frac{\sigma_{i j}}{r}\right)^{12}-\left(\frac{\sigma_{i j}}{r}\right)^{6}\right],
$$

where $\sigma$ is the minimum distance between monomers, roughly the monomer diameter and $\varepsilon$ is the well depth at the minimum of the potential. These parameters are set to $\sigma=4.3 \AA$ and $\varepsilon=0.8066 \mathrm{Kcal} / \mathrm{mol}$ for the interaction between two PEO monomers. The indices $i j$ distinguish polymer-polymer (pp), polymer-substrate (ps) and substrate-substrate (ss). It should be noted that nearest neighbors do not interact via the LJ potential for PEO. Bonded particles, meaning nearest neighbors that are connected via a bond, are bonded by harmonic bonds with the potential,

$$
V_{\text {bond }}=K_{b}\left(b-b_{0}\right)^{2},
$$

where the bond force constant is $K_{b}=20.32 \mathrm{Kcal} / \mathrm{mol} \cdot \AA^{2}$ and the equilibrium bond length is $b_{0}=3.30 \AA$. There is also an angle potential,

$$
V_{\text {angle }}=K\left[\cos (\theta)-\cos \left(\theta_{0}\right)\right]^{2}
$$

similar to the bond potential constants, where $K=10.16 \mathrm{Kcal} / \mathrm{mol}$ is a force constant and $\theta_{0}=130^{\circ}$ is the preferred equilibrium angle between adjacent monomers. A dihedral 
potential may also be defined by,

$$
V_{\text {dihedral }}(\phi)=\sum_{i=1}^{m} K_{i}\left(1+\cos \left(n_{i} \phi-d_{i}\right)\right)
$$

where $m=4$, so there are four $K, n$ and $d$ values which are listed in Table 1 .

Table 1: Dihedral potential parameters for $K, n$, and $d$ from Eq. 5

\begin{tabular}{|c|c|c|c|}
\hline$i$ & $\mathrm{~K}(\mathrm{Kcal} / \mathrm{mol})$ & $\mathrm{n}$ & $\mathrm{d}\left({ }^{\circ}\right)$ \\
\hline 1 & 0.46845 & 1 & 180 \\
\hline 2 & 0.04302 & 2 & 0 \\
\hline 3 & 0.07887 & 3 & 0 \\
\hline 4 & 0.02868 & 4 & 0 \\
\hline
\end{tabular}

Ref. ${ }^{1}$ provides further details on the potentials used in this model.

\section{PMMA}

The non-bonded beads interact with the same shifted LJ potential as Eq. 1, where the potential is shifted to have a smooth cut-off between $r_{1}=12.0 \AA$ and $r_{c}=15.0 \AA$. For the LJ potential, Eq. 2, there are six defined parameters: $\varepsilon_{A A}, \varepsilon_{B B}, \varepsilon_{A B}, \sigma_{A A}, \sigma_{B B}$ and $\sigma_{A B}$, where A represents the backbone bead and B is the side-group bead.

Table 2: Parameters of Lennard-Jones Potential for PMMA

\begin{tabular}{ccc}
\hline \hline & & \\
Bead & $\varepsilon_{i j}(\mathrm{Kcal} / \mathrm{mol})$ & $\sigma_{i j}(\AA)$ \\
\hline & & \\
$\mathrm{AA}$ & 0.5 & 5.5 \\
$\mathrm{BB}$ & 1.5 & 4.42 \\
$\mathrm{AB}$ & 0.866 & 4.96 \\
\hline \hline
\end{tabular}

The bonded particles interact via the same harmonic bond as PEO (Eq. 3), with spring constants $K_{b, A A}=105.0 \mathrm{Kcal} / \mathrm{mol} \cdot \AA^{2}, K_{b, A B}=39.86 \mathrm{Kcal} / \mathrm{mol} \cdot \AA^{2}$ and bond lengths $b_{0, A A}=$ $2.735 \AA$ and $b_{0, A B}=3.658 \AA$. 
The angle potential has two different forms, one for the AAA-angle and another for the AAB-angle. The AAA-angle potential is defined by the relation:

$$
V_{\text {angle }, A A A}(\theta)=-k_{b} T \ln \left[a_{1} \exp \left(-\frac{\theta-\theta_{1}}{b_{1}}\right)^{2}+a_{2} \exp \left(-\frac{\theta-\theta_{2}}{b_{2}}\right)^{2}\right],
$$

where $a_{1}=2.294 \times 10^{-2}, b_{1}=9.493^{\circ}, \theta_{1}=121.0^{\circ}, a_{2}=4.367 \times 10^{-3}, b_{2}=6.210^{\circ}$, and $\theta_{2}=158.5^{\circ}$. The AAB-angle potential equals,

$$
V_{\text {angle }, A A B}(\theta)=k_{2}\left(\theta-\theta_{0}\right)^{2}+k_{3}\left(\theta-\theta_{0}\right)^{3}+k_{4}\left(\theta-\theta_{0}\right)^{4},
$$

where $\theta_{0}=96.85^{\circ}, \mathrm{k}_{2}=9.881 \mathrm{Kcal} / \mathrm{mol} \cdot \mathrm{rad}^{2}, \mathrm{k}_{3}=-15.12 \mathrm{Kcal} / \mathrm{mol} \cdot \mathrm{rad}^{3}$, and $\mathrm{k}_{4}=6.589$ $\mathrm{Kcal} / \mathrm{mol} \cdot \mathrm{rad}^{4}$.

Similarly, the dihedral angle must be separated into two forms, one for the BAAB dihedral and another for the AAAA dihedral. The potential of the AAAA dihedral is described by,

$$
V_{\text {dihedral }, A A A A}(\phi)=\sum_{k=1}^{5} A_{k} \cos ^{k-1}(\phi)
$$

where the parameters $A_{1}-\mathrm{A}_{5}$ are the following:

\section{Table 3: Parameters for PMMA AAAA Dihedral Potential}

\begin{tabular}{ccccc}
\hline \hline \multicolumn{6}{c}{ AAAA Dihedral Parameters (Kcal/mol) } \\
\hline \multicolumn{6}{c}{$\mathrm{A}_{2}$} & $\mathrm{~A}_{3}$ & $\mathrm{~A}_{4}$ & $\mathrm{~A}_{5}$ \\
$\mathrm{~A}_{1}$ & $\mathrm{~A}_{2}$ & \\
4.380 & 0.8739 & -0.3571 & -0.2774 & 0.09312 \\
\hline \hline
\end{tabular}

The BAAB dihedral angle potential has the same form as the AAAA potential with different coefficients,

This model was developed by Hsu et al. and for more detail on how this model was parameterized refer to Ref. 2 . 


\section{Table 4: Parameters for PMMA BAAB Dihedral Potential}

\begin{tabular}{|c|c|c|c|c|}
\hline \multicolumn{5}{|c|}{ BAAB Dihedral Parameters (Kcal/mol) } \\
\hline $\begin{array}{c}\mathrm{A}_{1} \\
4.519\end{array}$ & $\begin{array}{c}\mathrm{A}_{2} \\
-0.8859\end{array}$ & $\begin{array}{c}\mathrm{A}_{3} \\
-1.692\end{array}$ & $\begin{array}{c}\mathrm{A}_{4} \\
0.5625\end{array}$ & $\begin{array}{c}\mathrm{A}_{5} \\
0.9562\end{array}$ \\
\hline
\end{tabular}

\section{Substrate interaction strengths}

The interaction strength between the polymer beads and the substrate is calculated by relating the components of the surface tension and the work of adhesion for the materials composing the substrate and polymer film, specifically

$$
\frac{\varepsilon_{s p}}{\varepsilon_{p p}}=\frac{\sqrt{\gamma_{\text {sub }}^{d} \gamma_{\text {poly }}^{d}}+\sqrt{\gamma_{\text {sub }}^{p} \gamma_{\text {poly }}^{p}}}{\gamma_{\text {poly }}}
$$

where the surface tension is $\gamma=\gamma^{d}+\gamma^{p}$. $^{3,4}$ The superscripts $d$ and $p$ refer to the dispersion and polar components of surface tension, respectively. The subscripts sub and poly refer to the substrate and polymer, respectively. We use experimental values of surface energy to have realistic interactions between the polymers and the substrate, tabulated in Table 5 .

Table 5: Surface energy

\begin{tabular}{cccc}
\hline \hline Materials & $\begin{array}{l}\text { Experimental Surface Energies } \\
\text { total }\left(\mathrm{mJ} \cdot \mathrm{m}^{2}\right)\end{array}$ & $\begin{array}{l}\text { Surface energy } \\
\text { polar }\left(\mathrm{mJ} \cdot \mathrm{m}^{2}\right)\end{array}$ & $\begin{array}{c}\text { Surface energy } \\
\text { dispersive }\left(\mathrm{mJ} \cdot \mathrm{m}^{2}\right)\end{array}$ \\
\hline $\mathrm{SiO}_{2} / \mathrm{Si}^{5}$ & 44.68 & 24.69 & 19.99 \\
$\mathrm{PMMA}^{6}$ & 40.20 & 35.80 & 4.40 \\
$\mathrm{PEO}^{7}$ & 42.90 & 30.90 & 12.00 \\
\hline \hline
\end{tabular}

For PMMA, the average non-bonded polymer-polymer interaction strength $\varepsilon_{p p}$ is thus given by $\varepsilon_{p p}=\left(\varepsilon_{A A}+\varepsilon_{B B}+2 \varepsilon_{A B}\right) / 4=0.933 \mathrm{Kcal} / \mathrm{mol}$. We then find the average interaction strength between PMMA and substrate, $\varepsilon_{s p}=0.8627 \mathrm{Kcal} / \mathrm{mol}$ using Eq. 9. Next, the non-bonded interaction strengths between each type of bead and the substrate particle are 
evaluated by

$$
\frac{\varepsilon_{s p}}{\varepsilon_{p p}}=\frac{\varepsilon_{s X}}{\varepsilon_{X X}}
$$

where $X \in\{A, B\}$. Lorentz-Berthelot rules are used to obtain the Lennard-Jones interaction parameters between the backbone and side-group beads. We tabulate the final non-bonded interaction parameters in Table 1 of the main text.

\section{Pair Correlation Function}

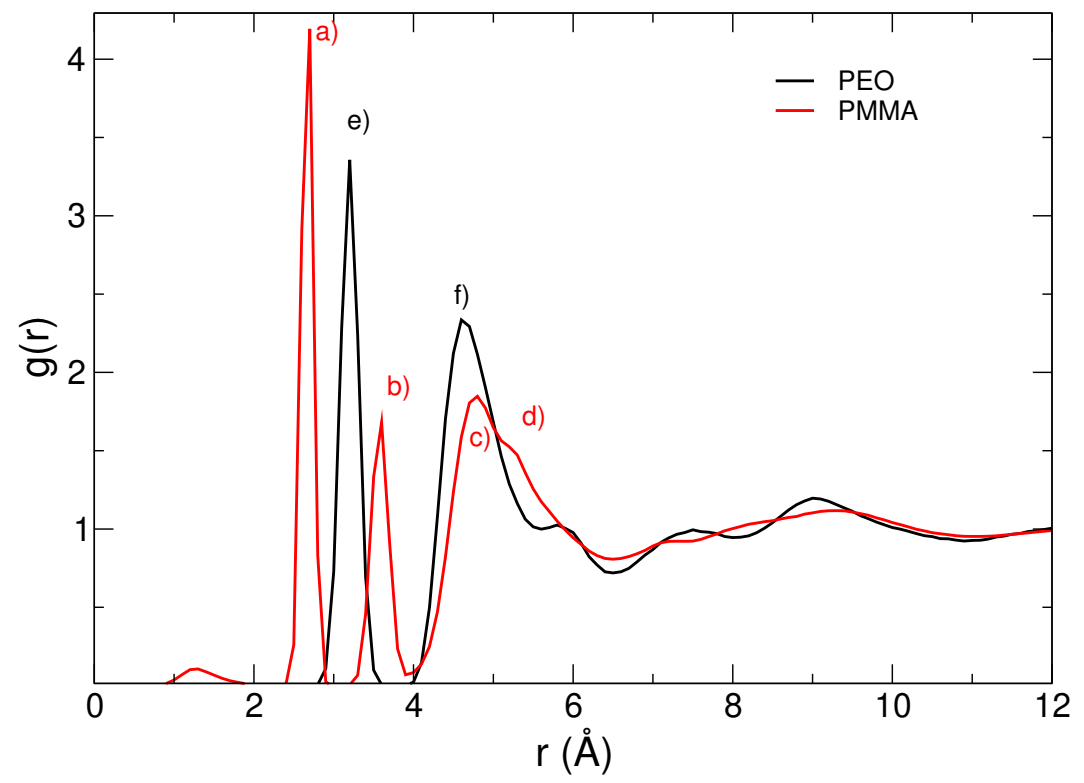

Figure S2: The pair correlation function, $g(r)$, of bulk PEO and PMMA where $\mathrm{r}$ is the distance between a pair of atoms, measured in $\AA$. The labeled peaks of interest for PMMA (in red) are $a$ ) the backbone-backbone bond length, $b$ ) is the backbone-side-group bond length, $c$ ) is the backbone-side-group pair interaction length and $d$ ) is the backbone-backbone pair interaction length. For PEO, the peak $e$ ) is the from the bonded interactions and $f$ ) is from the pair interactions.

From the pair correlation function $g(r)$ and the structure factor $S(q)$, the presence of the side-group and the different potential interaction lengths affect the overall structure in bulk. Since these parameters are unchanged under confinement, this structure remains the same in film. The persistence length is nearly twice as long for PMMA than for PEO, indicating PMMA has a more rigid backbone. The difference in density profiles suggests that PEO has 
a higher packing efficiency since the chains are allowed to pack closer and more densely near the attractive substrate.

\section{Structure Factor $S(q)$}

While the radial distribution function is straightforward to compute for simulations, it is only indirectly measured experimentally. Therefore, looking at the structure factor, $S(q)$, which can be directly measured from scattering experiments such as, X-ray or neutron diffraction Since $S(q)$ is a Fourier transform of $g(r)$, it more plainly reflects periodicities in the intramolecular packing. As such, $S(q)$ is particularly helpful to assess the degree of crystallization. The structure factor is defined as:

$$
S(q)=\frac{1}{N}\left\langle\rho(q) \rho^{*}(q)\right\rangle
$$

where $q$ is a wave vector and $\rho(q)=\sum_{j=1}^{N} e^{-i \mathbf{q} \cdot \mathbf{r}_{j}}$ is a Fourier transform of the density when particles are treated as points. The structure factor is a Fourier transform of $g(r)$ so the same reasoning follows that as temperature decreases, the peaks become sharper, which can be seen in Fig. S3 From this figure, we can see that the nearest neighbor periodicity is at

wave vector, $q=1.48 \AA^{-1}$ and $q=1.5 \AA^{-1}$ for PEO. These locations are significant since they are the $q$ values where dynamical correlations are usually measured.

\section{Persistence Length}

Chain flexibility is a key molecular feature that is known to affect shifts in glass transition temperature. To characterize the chain flexibility, we calculate the persistence length of PEO and PMMA in both bulk and films. To obtain the persistence length, we first evaluate the 

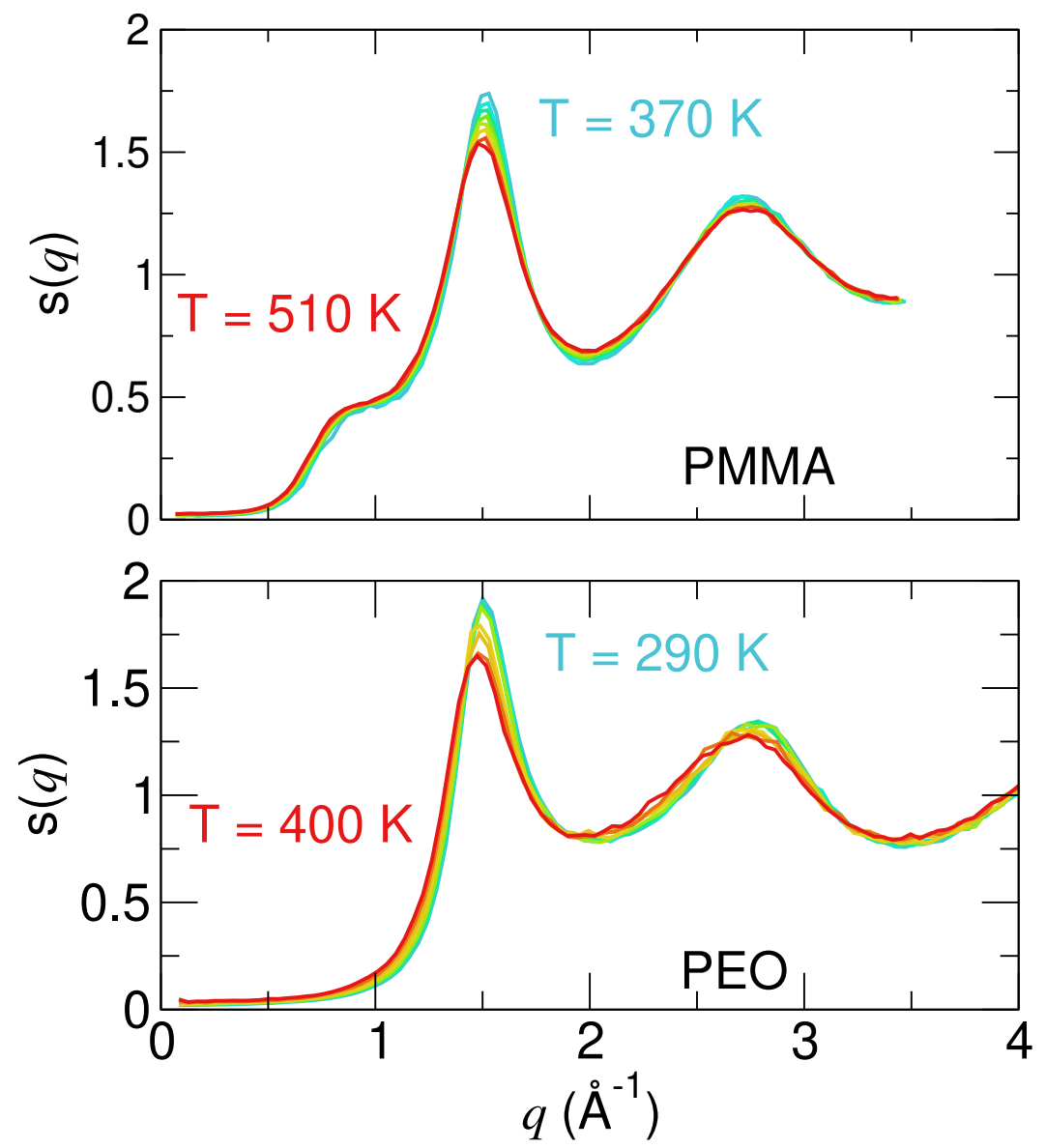

Figure S3: The structure factor at a variety of temperatures from low to high of bulk PEO and PMMA.

bond orientation correlation between any two backbone beads along the same chain,

$$
\langle\cos (\theta(s))\rangle=\langle u \overrightarrow{(r)} u(\overrightarrow{0})\rangle
$$

where $\vec{u}\left(r_{i}\right)=\overrightarrow{r_{i}}-\overrightarrow{r_{0}}$. By calculating this correlation function for a variety of temperatures in Fig. S4, we see that temperature weakly impacts the correlation between bond orientation and backbone separation.

The behavior of $\langle\cos (\theta(s))\rangle$ is typically exponential which allows for a simple definition of persistence length, $l_{p}$,

$$
\langle\cos (\theta(s))\rangle=\exp \left(-\frac{s}{l_{p}}\right),
$$


where $s$ is the backbone separation distance. Since this behavior is exponential, we extract the temperature-dependent persistence length at the separation distance where $\left\langle\cos \left(\theta\left(l_{p}^{\prime}\right)\right)\right\rangle=$ $1 / e$. Note that the $1 / e$ criterion is equivalent to $l_{p}$ from Eq. 13 obtained from the fit to $\langle\cos (\theta(s))\rangle$ mathematically, if the variation of $\langle\cos (\theta(s))\rangle$ is strictly exponential. We use the $1 /$ criterion to be consistent with the definition of $\tau$ from $F_{s}\left(q_{0}, t\right)$.
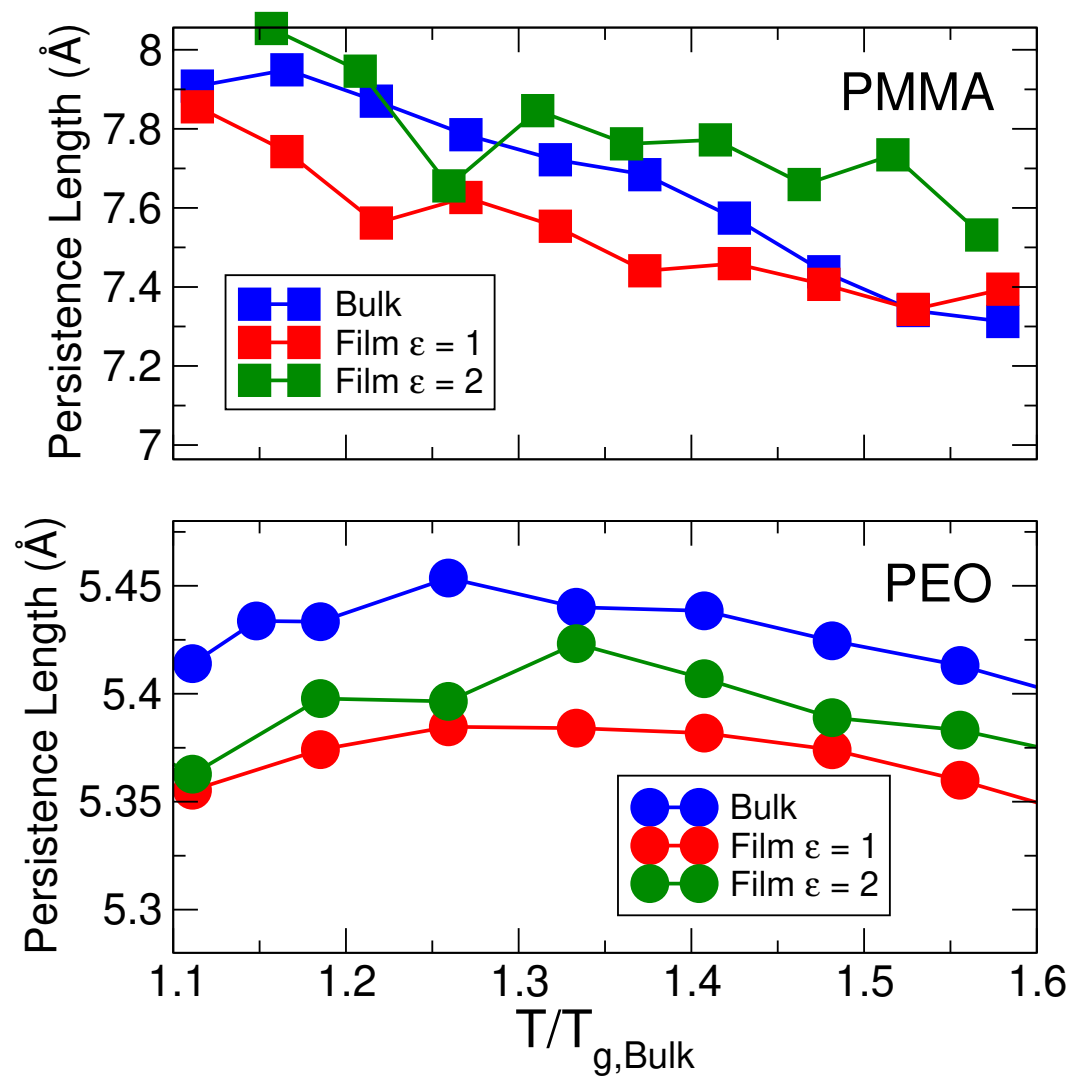

Figure S4: The persistence length measured in $\AA$ as a function of the normalized temperature $T / T_{g}$.

Since the persistence length is a weakly varying function of $T$, we quantify the flexibility of the chains by averaging the persistence length over the temperature range $T / T_{g, B u l k}=1.1$ to 1.5. From the average persistence lengths listed in Table 6, it is clear that PMMA is much stiffer than PEO. The number that follows \pm indicates the standard deviation of the persistence length. Our models produce persistence length that is reasonably close to the experimental values for PEO and PMMA, which are $4.1 \AA$ and $6.9 \AA$, respectively. ${ }^{8}$ 
Table 6: Table of average persistence lengths

\begin{tabular}{lccc} 
Material & Bulk $(\AA)$ & Film $\varepsilon=1(\AA)$ & Film $\varepsilon=2(\AA)$ \\
\hline & & & \\
PEO & $5.43 \pm 0.01$ & $5.37 \pm 0.01$ & $5.39 \pm 0.02$ \\
PMMA & $7.66 \pm 0.23$ & $7.54 \pm 0.16$ & $7.77 \pm 0.16$ \\
\hline \hline
\end{tabular}

\section{The Spatially-Resolved Incoherent Intermediate Scattering}

\section{Functions for PMMA and PEO}
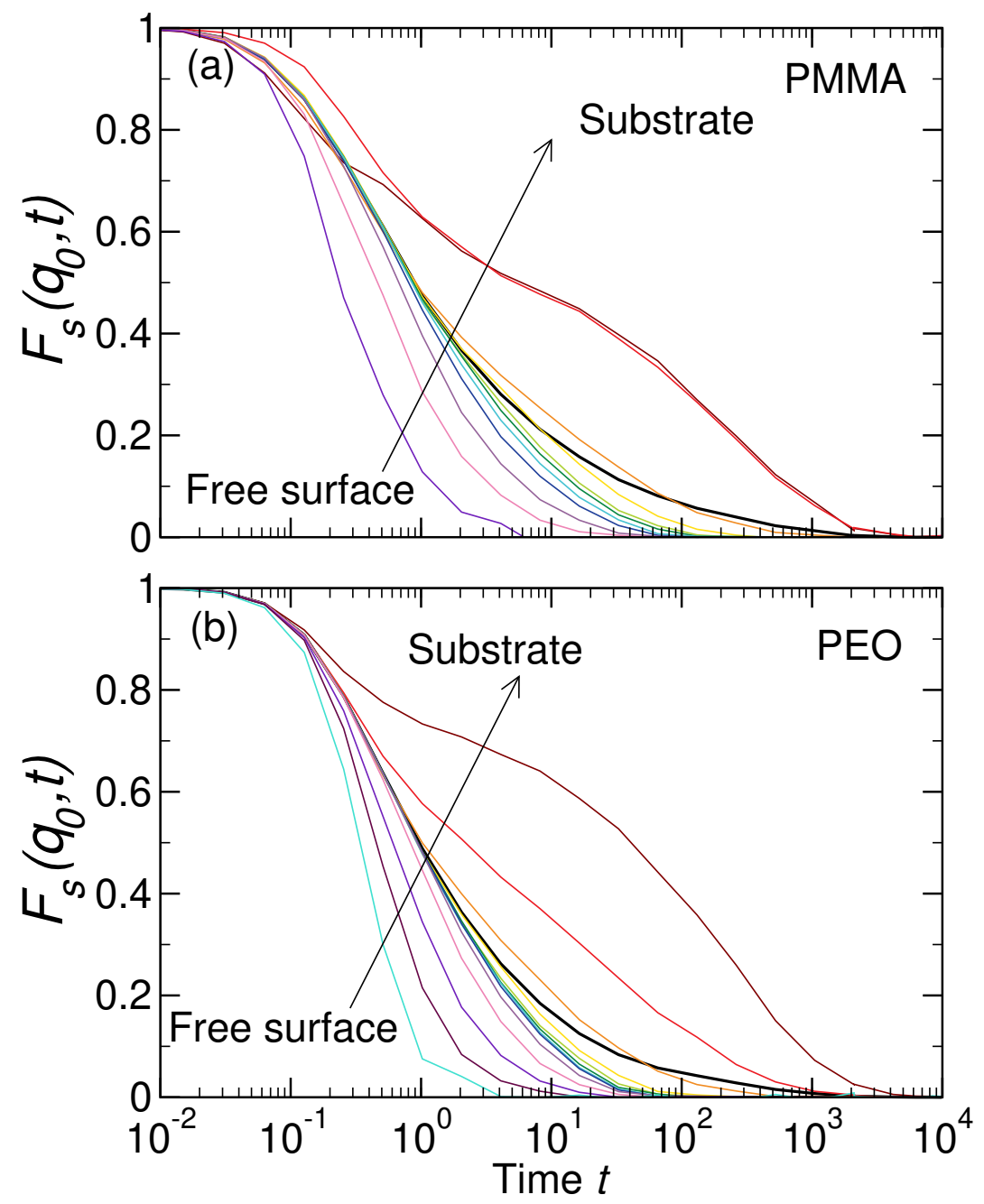

Figure S5: The incoherent intermediate scattering functions $F_{s}\left(q_{0}, t\right)$ for each monolayer of (a) PMMA and (b) PEO at $T \approx 1.6 T_{g}$ with enhanced substrate-polymer interaction strength. The thickness of each layer is approximately the diameter of the backbone bead. We also include $F_{s}\left(q_{0}, t\right)$ for th film as a whole (black curves) in each panel. 
We partition each bead into monolayers that are parallel to the substrate, based on their position at each time origin. We then evaluate $F_{s}\left(q_{0}, t\right)$ for each layer for both PMMA and PEO in Fig. S5. We see that the relaxation time of layers near the substrate is significantly slower than that near the free surface.

\section{The Dynamics of Backbone and Side-Chain for PMMA}
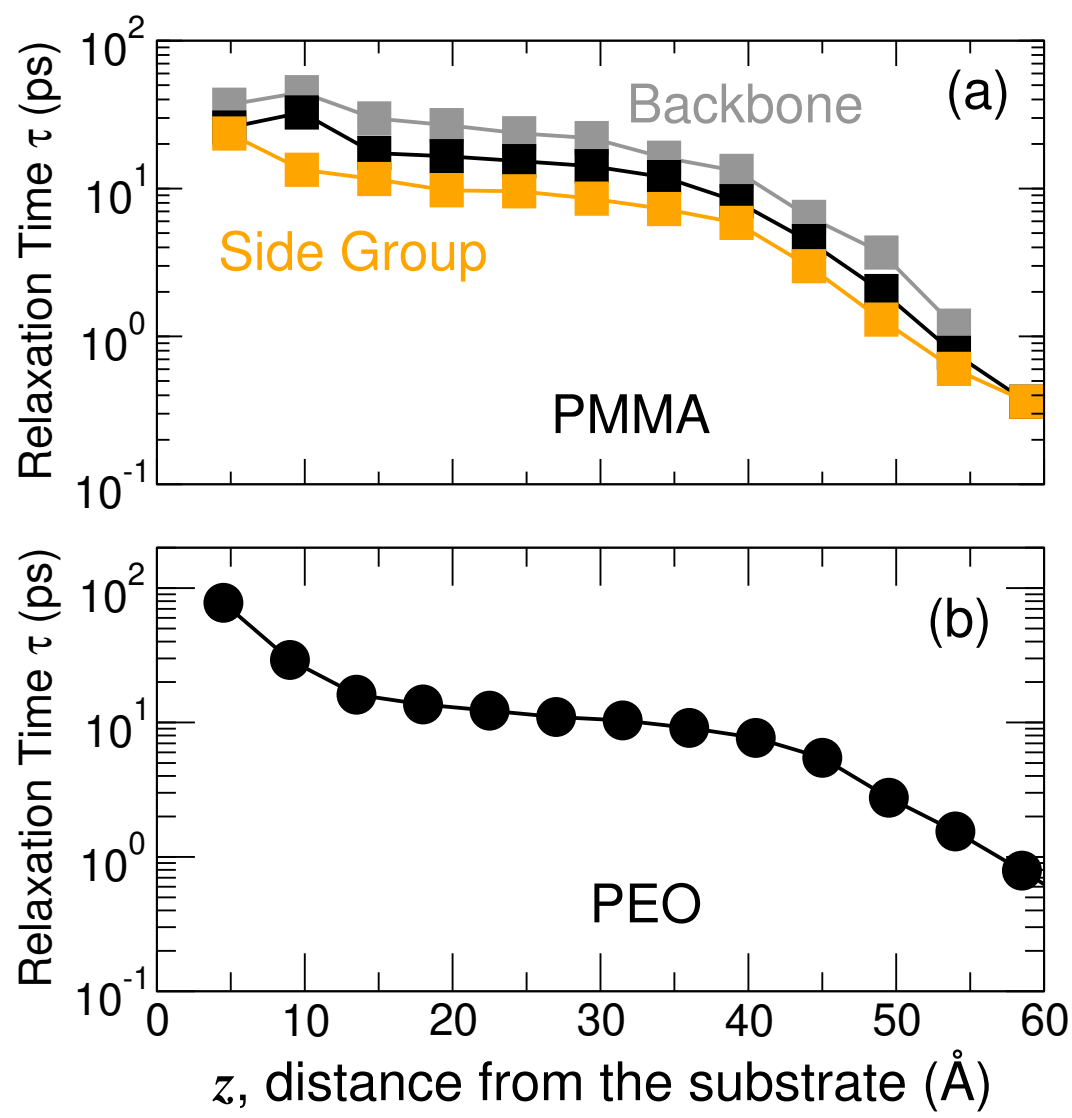

Figure S6: Evaluated at $T / T_{g}=1.4$, the relaxation time profile of PMMA (a) and PEO (b) supported by the natural substrate show how relaxation time varies as a function of distance from the substrate $z$.

For PMMA in panel $(a)$ of Fig. S6, the relaxation time is enhanced for the side-group beads within the first $4.5 \AA$ of the film. The increased crowding at this distance caused the relaxation time of the side-group beads to increase. As a consequence of the preferential organization, the backbone density decreases at $z=4.5 \AA$ but becomes significantly enhanced 
at $10 \AA$ resulting in a reduction in backbone relaxation time at $z=4.5 \AA$ but a large increase in $\tau$ at $z=10 \AA$. The combination of these two effect, drags the overall relaxation time down at $z=4.5 \AA$ but up at $z=10 \AA$. For PEO, since there is no competition between the side-group and backbone, the relaxation time profile follows the expected trend of steadily increasing as you approach the substrate from the interior. In regions where there is a normal organization amongst the backbone and side-group beads within the film, exemplary in the interior, the side-group bead relaxation is faster which supports what was seen in the mean-square displacements of the side-group and backbone.

\section{Characterization Film Relaxation Time}

As shown in Fig. S7 for PMMA, we can fit $F_{\mathrm{s}}\left(q_{0}, t\right)$ for the film as whole using the functional form

$$
F_{\mathrm{s}}\left(q_{0}, t\right)=(1-A) e^{-\left(t / \tau_{s}\right)^{3 / 2}}+\left(A-A_{b}\right) e^{-(t / \tau)^{\beta}}+A_{b} e^{-\left(t / \tau_{b}\right)^{\beta_{b}}}
$$

used in previous studies, ${ }^{9,10}$ where $\tau$ is the $\alpha$ relaxation time, $\tau_{b}$ is the bound relaxation time near the substrate. We see that the resulting $\tau$ and $\tau_{b}$ from Eq. 14 are nearly the same as $\tau$ obtained using the $1 / e$ criterion from the $F_{s}\left(q_{0}, t\right)$ of the film as a whole and the substrate layer, respectively. 

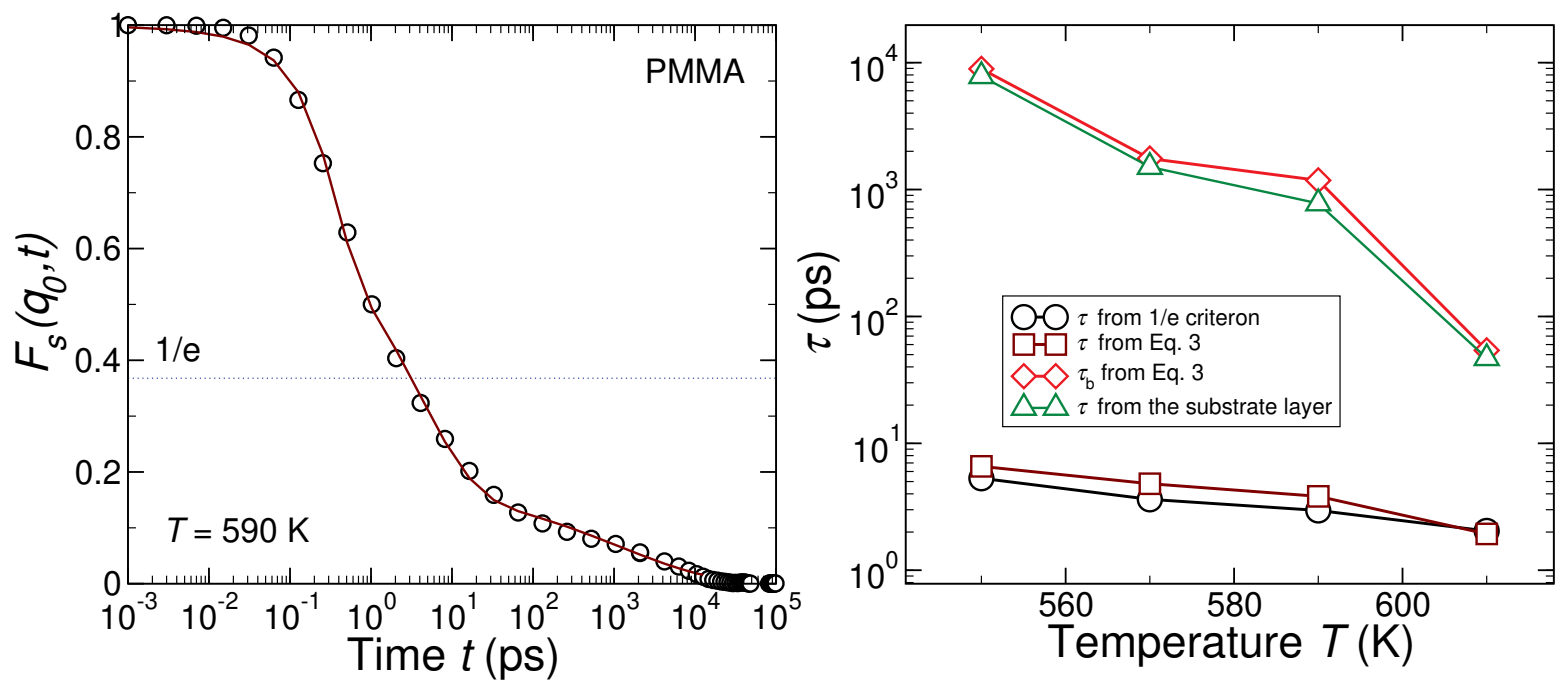

Figure S7: (Left) The intermediate scattering function $F_{s}\left(q_{0}, t\right)$ for supported PMMA film with enhanced substrate attraction. The symbols are data and line is the fit to the data using Eq. 14. (Right) The relaxation time of PMMA film with enhanced substrate attraction as a function of the temperature $T$.

\section{Fragility of Bulk PMMA and PEO}

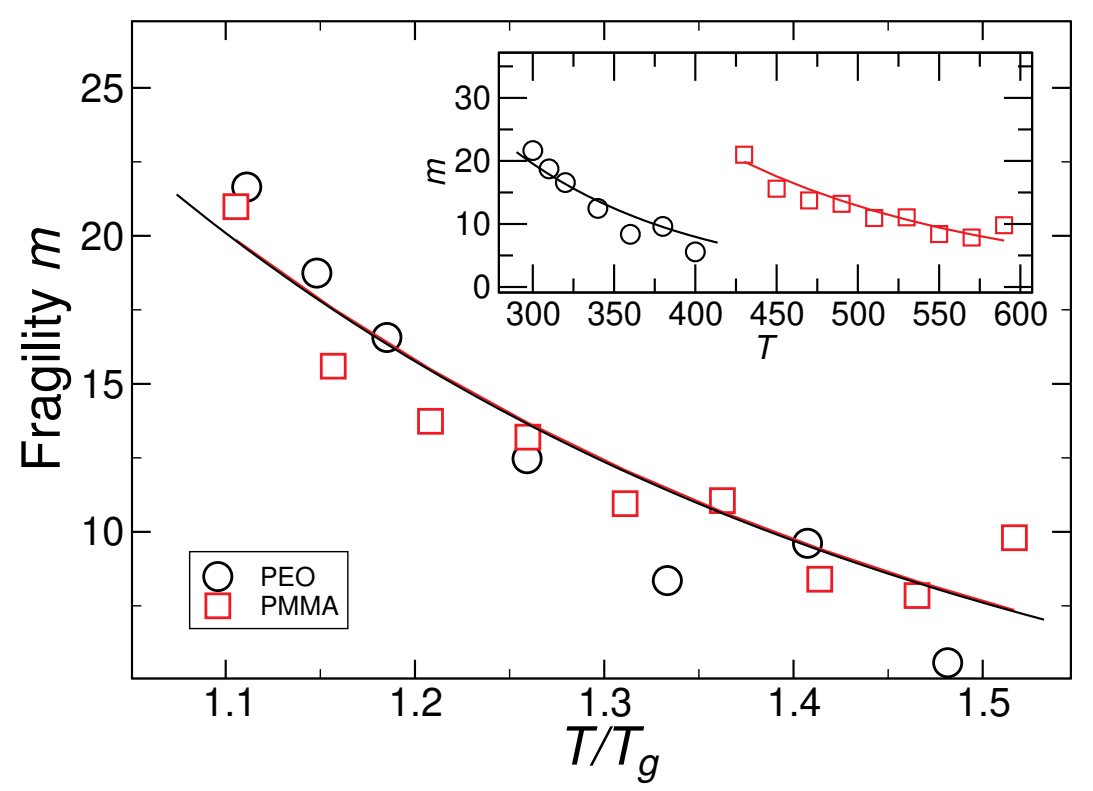

Figure S8: The temperature dependence of fragility for bulk PMMA and PEO, normalized by their respective $T_{g}$. The inset shows the unnormalized fragility $m$. 
The fragility at $T_{g}$ is commonly defined by,

$$
m\left(T_{g}\right)=\left.\frac{1}{T_{g}} \frac{\mathrm{d} \ln \tau}{\mathrm{d} 1 / T}\right|_{T_{g}},
$$

which generalizes to a temperature dependent fragility simply by evaluating the derivative at temperature $T$. Fig. S8 shows the $T$ dependent fragility evaluated directly from $\tau$ with no fitting. The results are somewhat noisy due to the numerical derivative that is required, but it is clear that the overall fragility of the two systems cannot be readily distinguished.

\section{The Interaction Energy Between Each Polymer and Substrate}

The interaction energy between polymers and the substrate are $-0.416 \mathrm{kcal} / \mathrm{mole}$ for PMMA supported by the natural substrate at $T=430 \mathrm{~K},-0.984 \mathrm{kcal} / \mathrm{mole}$ for PMMA supported by the enhanced substrate at the same $T,-0.320 \mathrm{kcal} /$ mole for PEO supported by the the natural substrate at $T=300 \mathrm{~K},-0.741 \mathrm{kcal} /$ mole for $\mathrm{PEO}$ supported by the enhanced substrate at the same $T$.

\section{Compressibility}

The compressibility of PEO and PMMA is shown in Fig. S9.

\section{References}

(1) Hwankyu Lee, A. H. d. V., et al. A Coarse-Grained Model for Poly-ethylene Oxide and Polyethylene Glycol: Conformation and Hydrodynamics. Journal of Physical Chemistry B 2009, 113, 13186-94, DOI: https://doi.org/10.1021/jp9058966.

(2) Hsu, D. D.; Xia, W.; Arturo, S. G.; Keten, S. Systematic Method for Thermomechanically Consistent Coarse-Graining: A Universal Model for Methacrylate- 


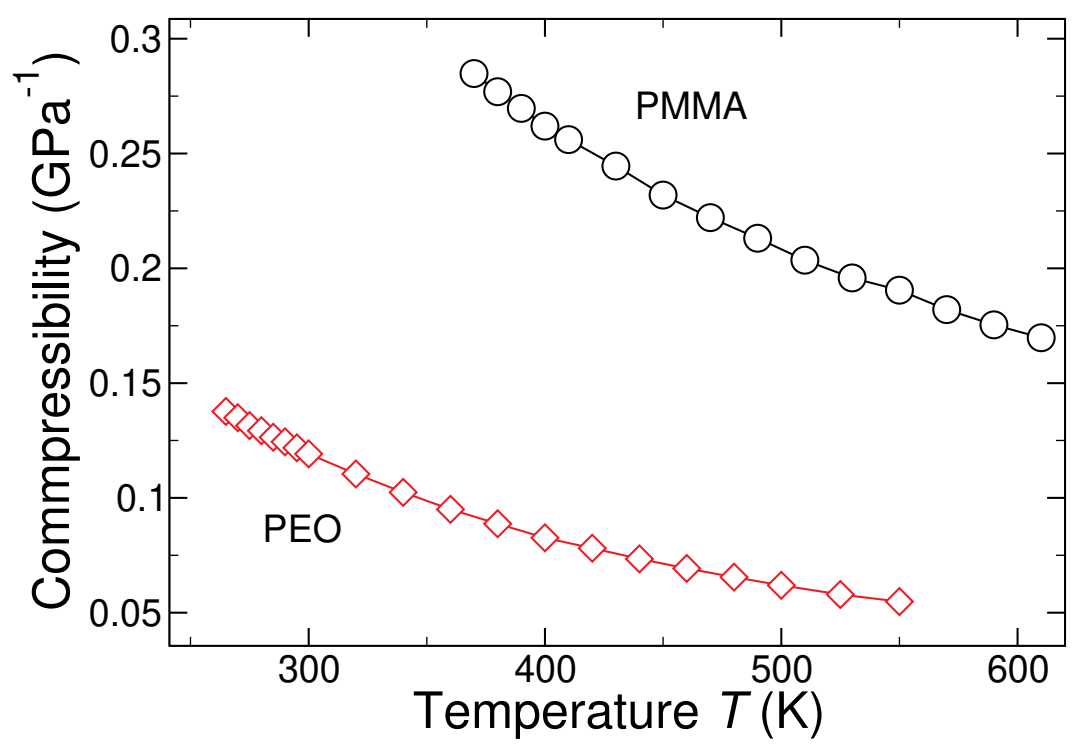

Figure S9: The compressibility for PMMA and PEO.

Based Polymers. Journal of Chemical Theory and Computation 2014, 10, 2514-2527, DOI: $10.1021 /$ ct500080h, PMID: 26580772.

(3) Good, R. J.; van Oss, C. J. Modern approaches to wettability; Springer, 1992; pp 1-27.

(4) Surface free energy-theory and calculations. https://cdn2.hubspot.net/hubfs/ 516902/Pdf/Attension/Tech\%20Notes/AT-TN-04-Surface-free-energy-theory . pdf.

(5) Annamalai, M.; Gopinadhan, K.; Han, S. A.; Saha, S.; Park, H. J.; Cho, E. B.; Kumar, B.; Patra, A.; Kim, S.-W.; Venkatesan, T. Surface energy and wettability of van der Waals structures. Nanoscale 2016, 8, 5764-5770.

(6) Natarajan, B.; Li, Y.; Deng, H.; Brinson, L. C.; Schadler, L. S. Effect of interfacial energetics on dispersion and glass transition temperature in polymer nanocomposites. Macromolecules 2013, 46, 2833-2841.

(7) Solid surface energy data (SFE) for common polymers. 2018 (accessed August 21, 2020); http://www. surface-tension.de/solid-surface-energy.htm. 
(8) Hiemenz, P.; Lodge, T. Polymer Chemistry; CRC Press, 2007.

(9) Starr, F. W.; Douglas, J. F.; Meng, D.; Kumar, S. K. Bound layers "cloak" nanoparticles in strongly interacting polymer nanocomposites. ACS nano 2016, 10, 10960-10965.

(10) Zhang, W.; Douglas, J. F.; Starr, F. W. Effects of a "bound" substrate layer on the dynamics of supported polymer films. The Journal of Chemical Physics 2017, 147, 044901. 